\title{
BMJ Open effect of physical activity on independent living ability among community-dwelling elderly in urban areas of Liaoning Province in China: a population-based study
}

\author{
Bowen Wang, ${ }^{1,2}$ Yijiao Wu, ${ }^{1}$ Tianjiao Zhang, ${ }^{1}$ Jinsong Han, ${ }^{1}$ Lianzheng $\mathrm{Yu},{ }^{3}$ \\ Wei Sun (iD ${ }^{1}$
}

To cite: Wang B, Wu Y, Zhang T, et al. effect of physical activity on independent living ability among communitydwelling elderly in urban areas of Liaoning Province in China: a populationbased study. BMJ Open 2019;9:e023543. doi:10.1136/ bmjopen-2018-023543

- Prepublication history and additional material for this paper are available online. To view these files, please visit the journal online (http://dx.doi org/10.1136/bmjopen-2018023543).

BW and YW contributed equally.

Received 11 April 2018

Revised 06 June 2019

Accepted 26 September 2019

Check for updates

(C) Author(s) (or their employer(s)) 2019. Re-use permitted under CC BY-NC. No commercial re-use. See rights and permissions. Published by BMJ.

For numbered affiliations see end of article.

Correspondence to

Professor Wei Sun;

wsun@cmu.edu.cn

\section{ABSTRACT}

Objective Independent living ability is crucial for the elderly; however, its assessment performed in China employs various scales and lacks risk factors, especially behavioural and social-psychological factors, which could be improved through health education. This study aimed to assess the independent living ability of communitydwelling elderly in urban areas of China and to identify the association with behavioral and social-psychological factors.

Design A population-based study using random cluster sampling method was performed in two core cities in Liaoning Province during March-November 2012.

Study population 3686 individuals who were $>=65$ years old, had no dementia and cognitive impairment, had lived in the sampled communities for $>=5$ years and could take part in the study were enrolled.

Main outcome measures Interviews were conducted to collect information of independent living ability, indicated by the instrumental activity of daily living (IADL), behavioural factors, social-psychological factors and confounders (demographic characteristics and health status). The Mini-Mental State Examination was used to screen for cognitive impairment.

Results The independence rate was $46.3 \%$ in men and $41.1 \%$ in women. Structural equation modelling analysis showed that physical activity, drawn from taking a walk and doing exercise, had the strongest association with the IADL in both men $(-0.34$, SE $0.10)$ and women $(-0.33$, SE 0.11$)$. Falling in the last year and worrying about falling were also significantly associated with IADL. The effects of regular drinking, feeling lonely and participating in entertainment were significant in men.

Conclusion The independent living ability of the elderly in urban areas in Liaoning Province in China was at a low level. Physical activity was one of the important roles in both men and women; whereas the role of social-psychological factors only existed in men. Gender-specific healthcare and education to avoid sedentary life should be advocated for the elderly to maintain/improve their independent living ability.

\section{Strengths and limitations of this study}

- The Lawton Instrumental Activities of Daily Living (IADL) Scale was applied in this study, which has been used worldwide to indicate independent living ability of the elderly.

- The effects of behavioural and social-psychological factors that could be improved through health education in daily life were assessed among a Chinese population with adjustment for demographic characteristics and health status, whereas the measurements of smoking and regular drinking were broad and simplistic as the first attempt.

- Information bias was controlled as much as possible because the cognitive function of the elderly without dementia was further screened using the MiniMental State Examination.

- Quantitative assessment through scoring measured factors according to the $\mathrm{OR}$ values was conducted and the effects on IADL were confirmed by structural equation modeling analysis.

\section{INTRODUCTION}

A significant change in the age structure of the world's population has resulted from population ageing, ${ }^{1}$ and has become a global issue in the 21 st century. ${ }^{23}$ China is a nation with the largest ageing population, which accounts for one-fifth of the total elderly population worldwide. ${ }^{4}$ Indeed, it has been estimated that China will experience the most rapid rate of ageing in the world over the next 2-3 decades. ${ }^{1}$ Thus, how to cope with problems caused by population ageing has become particularly important for the development of China.

For the elderly, independence in daily life reduces nursing demands, decreases the consumption of medical resources and improves the quality of life. Even if the loss of independent living ability is inevitable for the 
elderly, maintaining and/or improving this ability, even for short time, can postpone the onset of dependency and reduce the enormous social burden. Thus, focusing on the independent living ability among the elderly prior to death or becoming ill is important.

Previous studies involving the independent living ability of the elderly have been conducted in developed countries. ${ }^{5-9}$ The factors associated with the independent living ability of the elderly have been shown to be demographic characteristics (age, gender, ethnicity, marital status, education and living arrangement), health status (chronic disease, visual ability and hearing ability), behaviour factors (smoking, alcohol consumption, taking a walk, doing exercise and falling risk) and social-psychological factors (social integration, depressive symptoms and social activity). Related studies have also been conducted in China: ${ }^{10-12}$ however, various scales were used to indicate independence. As a result, the rate of dependency ranged from $7.9 \%$ to $53.9 \%$. The risk factors were limited to demographic characteristics and chronic disease. As for behavioural and social-psychological factors, health education interventions for smoking, respiratory function exercise, diabetes-related activity, anxiety and family support had already been identified to improve health status of the elderly. ${ }^{13-15}$ Thus, these factors should also be studied from the perspective of independent living ability. Unfortunately, those educational risk factors in daily life were not considered in China. In addition, cognitive function screening was rarely considered in international and domestic studies, which was insufficient with respect to excluding information bias, especially for the aged population.

The purpose of this study was to conduct a population-based study to assess the independent living ability among the elderly without cognitive impairment in China, and to clarify the effects of behavioural and social-psychological factors with adjustment for demographic characteristics and health status. As a first attempt, we initially focused our attention on the elderly in urban areas. We believe our findings will provide help for the healthcare system to properly cope with population ageing.

\section{METHODS}

\section{Study area and population}

According to the China Yearbook, the income level in Liaoning Province is similar to the national average. Shenyang City and Anshan City are the core cities in Liaoning Province and represent the urban area according to the Liaoning Provincial Yearbook. Two districts in each city were randomly sampled and one community in each sampled district was randomly selected. People who were $>=65$ years of age, had never be diagnosed as dementia by physicians, lived in the local area for $>=5$ years and volunteered to take part in this study comprised the study population (a total of 4701 individuals). Investigators were recruited from graduate students who majored in medical epidemiology and trained for 2 weeks with the purpose of controlling respondent bias. Face-to-face interview was conducted between March and November 2012. A total of 4057 participants completed $80 \%$ and more of interview and were identified as effective responses. Six hundred and forty-four responses were defined as dropouts because the participants had something to do, lost patient to complete the interview or declined to take part, although they agreed at the beginning. The effective response rate was $86.3 \%$. Cognitive function was further screened with the Mini-Mental State Examination (MMSE). Cognitive impairment was identified with consideration of education as follows: illiterate $<=17$, elementary $<=20$, middle $/$ high school $<=22$, junior college and over $<=23$. In the current study, 3686 individuals without cognitive impairment were enrolled as the subjects.

\section{Measurement of independent living ability}

The Lawton Instrumental Activities of Daily Living (IADL) Scale has been widely used to assess independent living ability of the elderly. ${ }^{16}$ It has been validated among elderly Chinese. ${ }^{17}$ The IADL Scale has eight items assessing the ability to use the telephone, shop for daily necessities, prepare meals, handle finances, do housework, take medications, do laundry and use public transportation. Responses to each item are coded as 1 (completely able), 2 (have some difficulty), 3 (need help) or 4 (unable). The total score ranges from 8 to 32 to represent the level of living ability. The higher the score, the worse the independent living ability. Independence is defined only if the total score is 8 .

\section{Measurements of behavioral factors, social-psychological factors and confounders}

Behavioural factors included the following seven items: (1) smoking; (2) regular drinking; (3) taking a walk; (4) doing exercise; (5) regular diet; (6) falling in the last year; and (7) worrying about falling. Smoking was measured with answers of 'Yes', 'Had ever' or 'Never'. Regular drinking was examined by asking 'Do you drink regularly (twice or more a week for more than a year)?'. The responses were categorised as 'Yes' or 'No'. Taking a walk and doing exercise (including Tai chi chuan, jogging, running, riding a bike, swimming and playing shuttlecock and table tennis) were divided into four groups on the basis of frequency: almost none; 1-2 times/week; 3-4 times/week; and almost every day. ${ }^{18}$ Regular diet was measured by asking 'Do you have breakfast, lunch and dinner on time and rarely skip meals?'. Falling in the last year was defined as 'Yes' or 'No'. Worrying about falling was defined as 'Yes' or 'No'.

Social-psychological factors consisted of the following four items: (1) feeling lonely; (2) participating in entertainment; (3) watching television often; and (4) filial piety. Feeling lonely was assessed according to an individual's perception and was categorised as 'Yes', 'Not clear' or 'No'. Information on participating in entertainment and watching television often was examined by asking 'Do 


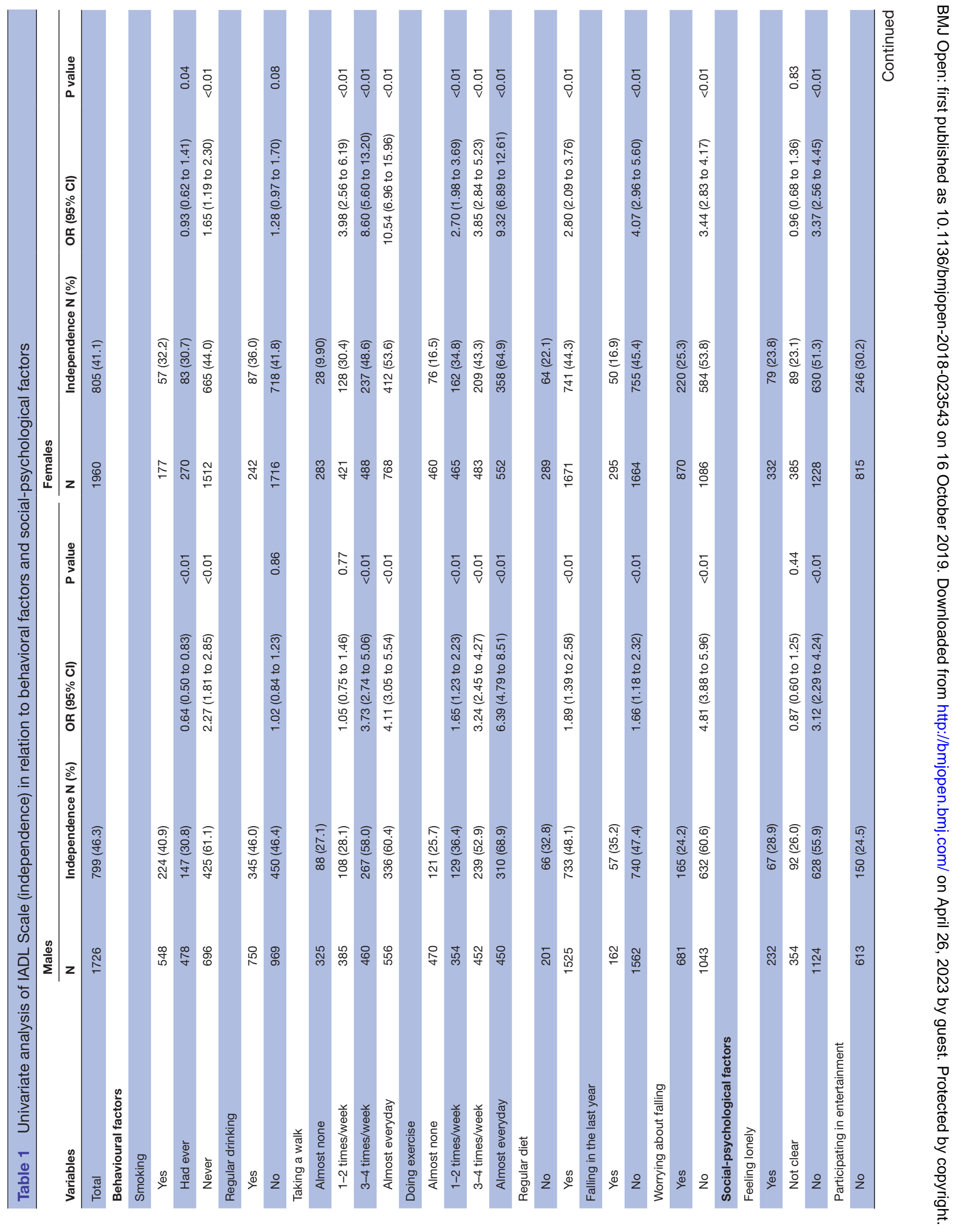


you always hang out and play cards, chess, mahjong, or some other entertainment? ${ }^{18}$, and 'Do you watch television almost every day?'. Filial piety is a prime virtue in Confucianism and advocated by Chinese traditional culture. The filial piety of children is expected to affect the health of the elderly. Thus, filial piety was included and assessed by asking 'How do you feel about your children's filial duty to you?'. The responses 'So so' and 'Bad' accounted for $8.8 \%$ and $0.4 \%$ of responses, respectively. These responses were combined into one group.

Confounders included factors of demographic characteristics and health status. Demographic characteristics included age, gender, ethnicity, marital status, living arrangement, pension and health insurance. For marital status, the responses of 'Single', 'Divorced' and 'Separation' were combined with 'Widow' as the 'Other' group because of the low response rate $(<5 \%)$. Health status included chronic disease, visual ability, ${ }^{8}$ hearing ability, ${ }^{8}$ daily sleep time, sleep quality and chewing ability. ${ }^{19}$ Chronic diseases were categorised as 'Present' if any diseases, such as hypertension, cardiovascular disease, diabetes, stoke, liver cirrhosis, emphysema, rheumatoid arthritis and chronic renal insufficiency, had been diagnosed. Visual ability was assessed to be 'Impaired' if participants could not read the newspaper clearly regardless of wearing reading glasses. Hearing ability was defined as 'Impaired' if voices for conversation or the volume for watching television had to be raised. For daily sleep time, the responses ' $<4$ hours' (2.7\%) and ' $>8$ hours' $(6.0 \%)$ were combined as one group. Sleep quality measured the difficulty in falling asleep or frequent awakening/ dreaming.

\section{Patient and public involvement}

Only community residents were involved as participants in this study. They received the written information about the conduct of the survey. However, they were not involved in the development of the research question and outcome measures, the recruitment of subjects and the undertaking of the study. After signing an informed consent, they were assessed for eligibility and data collection was conducted.

\section{Statistical analysis}

Among all independent variables, health insurance in both men and women and ethnicity in men were not considered in the data analysis because $>95 \%$ of the participants had the same response.

The association between IADL (independence) and each categorical variable was tested via a $\chi^{2}$ test. The ORs were obtained by Mantel-Haenszel estimates. Then, the quantitative values (scores) of these categorical variables were transformed according to the corresponding OR value of each group. Those quantitative values were used to perform structural equation modeling (SEM) to confirm the effect of factors measured on IADL score. Maximum likelihood estimates were selected to fit SEM. While performing SEM analysis, variables that were not 
Table 2 The description of scores of IADL and assessed factors

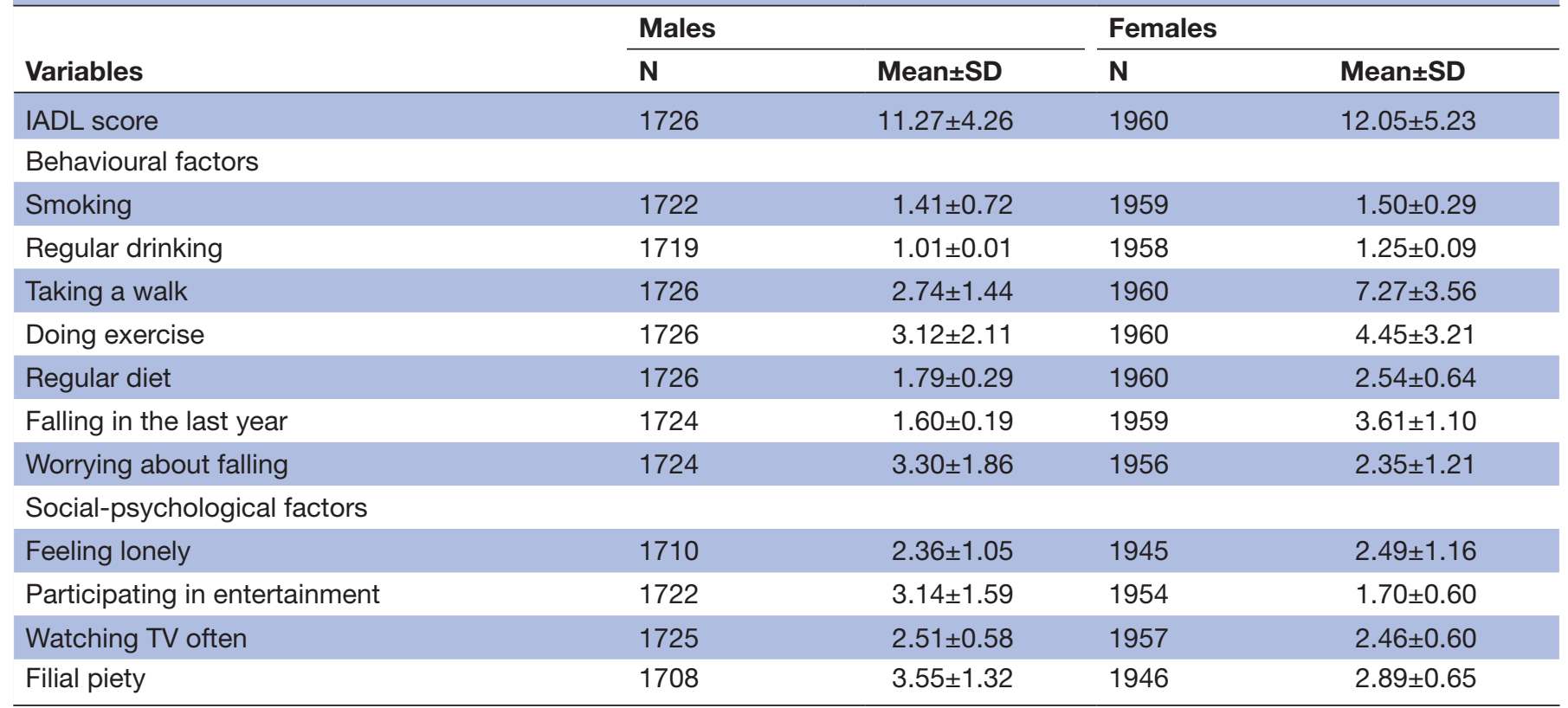

statistically significant were eliminated with the purpose to simplify the model. Elimination was conducted in the sequence of $p$ value, one at a time. When a variable was eliminated, if the goodness of fit became worse, the variable would remain in the model. In this study, no variable was found to decrease the goodness of fit during elimination. The simplified SEM model was shown and results are expressed as a standardised parameter estimate $(\beta)$ with SE representing the association between dependent variable and independent variables. The goodness of fit of model was indicated by the goodness-of-fit index (GFI), comparative fit index (CFI), Tucker-Lewis index (TLI) and root mean square error of approximation (RMSEA). The proposed values indicating high goodness of fit was GFI $>0.8$, CFI $>0.9$, TLI $>0.9$ and RMSEA $<0.08$.

The agreement between categorical variables was evaluated with the Kappa test. If the Kappa value was $>0.50$, these variables were considered in agreement. As for quantitative values, Pearson correlation was used to identify the collinear variables.

The missing data were not handled because the missing rate was $<5 \%$. But they were excluded when performing SEM model. SPSS for Windows (V.13.0) and AMOS (V.6.0) were used for all statistical analyses.

\section{RESULTS}

Among 3686 subjects, the independence in men and women was $46.3 \%$ and $41.1 \%$, respectively. A total of $43.5 \%$ of individuals were independent. In comparison to the results $(54.8 \%)$ obtained from urban areas in Shanghai $(\mathrm{n}=2944),{ }^{17}$ the level of independence was significantly lower $(\mathrm{p}<0.01)$. The results of univariate analysis of the IADL Scale (independence) are shown in table 1. Among the community-dwelling elderly in urban areas, the factors that were significantly correlated with the IADL included smoking, taking a walk, doing exercise, regular diet, falling in the last year, worrying about falling, feeling lonely, participating in entertainment, watching TV often and filial piety in both men and women. The distribution of IADL (independence) among demographic characteristics and health status is shown in online supplementary appendix A.

The average IADL scores were $11.27 \pm 4.26($ mean \pm SD) in men and $12.05 \pm 5.23$ in women. The items were scored according to the OR values. In this study, the correlations between 'taking a walk' and 'doing exercise' were 0.6252 in men and 0.5746 in women. Therefore, a latent variable, 'physical activity', drawn from these two variables was used, which was the same as confounders of 'marital status' and 'living arrangement' (r: 0.8074 in men and 0.6443 in women). A latent variable (family status) was obtained. The scores are shown in table 2. The scores of demographic characteristics and health status are shown in online supplementary appendix B.

SEM examinations for confirming the effects of behavioural and social-psychological factors were performed separately in men and women. The results are shown in figures 1 and 2. SEM analysis revealed that the latent variable (physical activity) had the highest association with the IADL score in both men and women. The standardised parameter estimate $(\beta)$ was -0.34 (SE: 0.10 ) in men and -0.33 (SE: 0.11 ) in women. In men, the IADL score was associated with feeling lonely, worrying about falling, regular drinking, falling in the last year and participating in entertainment, with adjustments for family status, age, chronic disease, chewing ability, daily sleep time, visual ability and sleep quality. The goodness of fit (GFI, CFI, TLI and RMSEA) was 0.917, 0.824, 0.763 


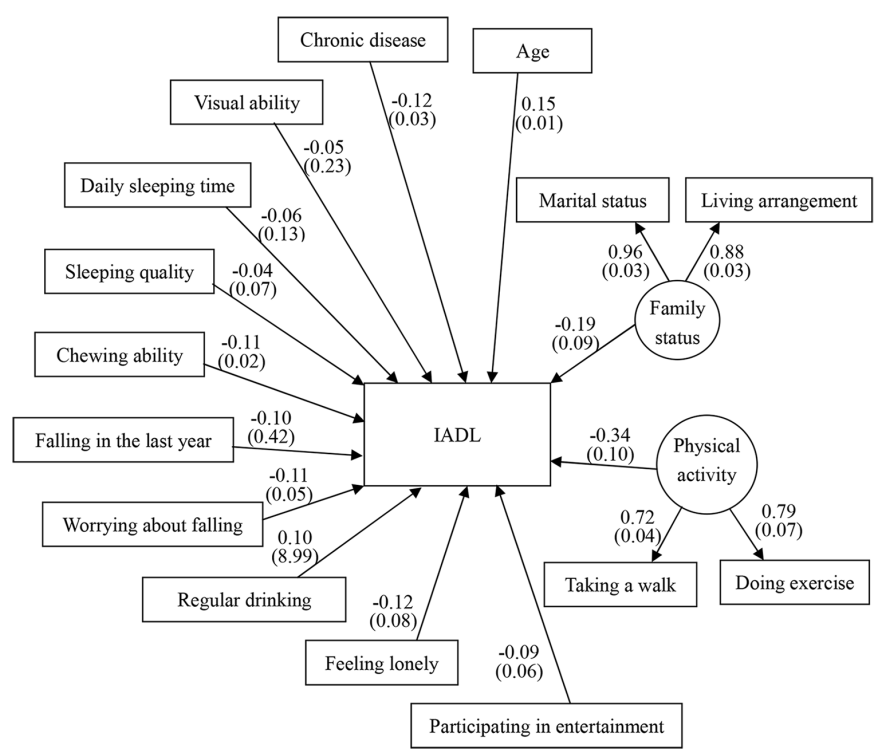

Figure 1 The SEM analysis for confirming the effects of behavioural factors and social-psychological factors in men $(n=1690)$. Data were shown as standardised estimate (SE).

and 0.083 , respectively. In women, the IADL score was associated with falling in the last year and worrying about falling, with adjustments for ethnicity, daily sleep time, family status, age, visual ability, chronic disease, pension and hearing ability. The goodness of fit (GFI, CFI, TLI and RMSEA) was $0.920,0.845,0.764$ and 0.093 , respectively.

\section{DISCUSSION}

Among the elderly in urban areas in Liaoning Province, the prevalence of independence in daily living ability was $43.5 \%$. The independence in daily living ability was significantly lower than the level $(54.8 \%)$ observed in the elderly living in urban areas in Shanghai, ${ }^{17}$ even though the average age of our study population (73.2 years) was much younger than the population in Shanghai (78.44 years). In addition, independent living ability in women $(41.1 \%)$ was lower than men $(46.3 \%)$, which was consistent with the results of the urban elderly in Suzhou ${ }^{12}$; however, the life expectancy of women (79.4) was longer than men (73.6) according to the China Yearbook, ${ }^{20}$ thus suggesting that more attention should be paid to maintaining independent living for a longer time in elderly women. These findings indicated that the independent living ability among the urban elderly in Liaoning province is still at a low level. In this study, the sample size was sufficiently large and information bias was controlled as much as possible because both dementia and cognitive function screening with MMSE were conducted. Although many potential reasons such as social desirability might play a significant role for information bias control, especially for the examination of alcohol consumption, cognitive impairment tended to be a prominent reason for information bias of the elderly. In addition, assessment on alcohol consumption in this study was focused on regular drinking habit instead of the exact consumption

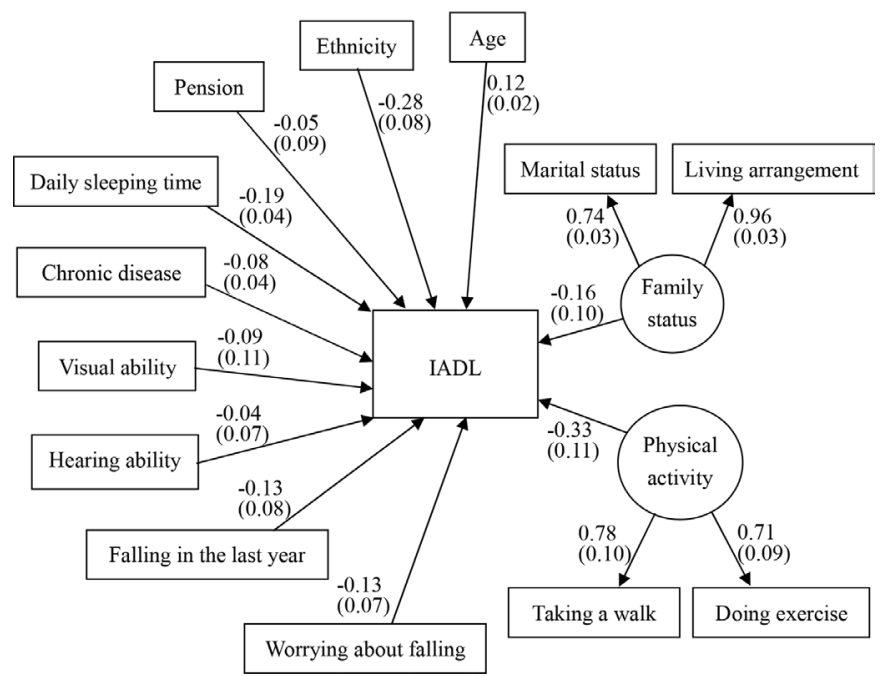

Figure 2 The SEM analysis for confirming the effects of behavioural factors and social-psychological factors in women $(n=1915)$. Data were shown as standardised estimate (SE).

volume, because the probability of heavy drink was low for the elderly. All these facts suggested that information bias could be controlled well and the generalisation of our conclusions could be increased. Our results suggested that maintaining/improving independent living ability of the elderly, especially women, should be an essential part of providing healthcare for the aged population.

While assessing the daily living ability of the elderly, the independent/dependent levels were the same as the definition of independence only from the viewpoint of health promotion. Because the IADL score indicates the level of independent living ability, the IADL score tended to be more valuable to quantitatively assess the associations between independent living ability and risk factors reported. Thus, the SEM model was performed; however, the variables entered in SEM model should be quantitative data, which became an obstacle for the assessment of categorical items. Thus, quantitative transformation according to OR value was used to overcome this obstacle. Even if CFI and TLI did not achieve the target value of 0.9 , the values were still over 0.7. As for RMSEA, the values for both men and women models were slightly over the target value of 0.08 , but still lower than 0.1. According to the standards recommended by Steiger, ${ }^{21}$ the goodness of fit of the SEM model was acceptable. Thus, we drew our conclusions based on the results of the SEM models.

With respect to the behavioural factors, physical activity was reported to maintain physical health of the elderly and prevent the elderly from acquiring mental diseases. ${ }^{22}$ In our study, taking a walk and doing exercise were considered to measure physical activity among the elderly; however, due to the high agreement between walking and doing exercise, previous studies have usually selected one item to enter into the multiple model while assessing their effects. In this study, the kappa value between taking a walk and doing exercise was also high (0.546). Of note, 
we are not satisfied with this type of adjustment because taking a walk and doing exercise require different intensities of activity. This kind of assessment was expected to weaken the effect of physical activity. Thus, we used the latent variable, physical activity, to represent the effects of both taking a walk and doing exercise. As a result, physical activity was shown to have the strongest association with IADL in both men and women. Although health status was reported to be crucial for the quality of life of the elderly in our previous study ${ }^{18}$ and the presence of chronic disease was well-documented as the basis of any disorder, ${ }^{23}{ }^{24}$ the associations with the IADL score were weaker than physical activity. We also compared the effect of the latent variable with the effects of original observed variables. When taking a walk was entered into the model instead of physical activity, the parameter estimate was lower $(-0.233$ in men and -0.228 in women) in comparison to physical activity, and the same as doing exercise $(-0.253$ in men and -0.244 in women). These results conversely proved that the previous adjustment did weaken the effect of physical activity. Furthermore, we found that the more frequent the elderly performed physical activity, the stronger the effect on the independent living ability. Thus, any type of activity should be advocated for the independence of the elderly regardless of health status.

With respect to behavioural factors, falling in the last year and worrying about falling were also associated with independent living ability in both men and women. The experience of falling in the last year may be an indicator for the decline of muscle strength, coordination, balance and flexibility, ${ }^{25}$ which tended to be a potential threat for fractures and becoming bedridden among the elderly. ${ }^{26}$ Furthermore, because of the significant correlation with visual ability ( 0.178 in men and 0.191 in women), the effect on health status also increased the possibility to affect the independent living ability of the elderly. Indeed, worrying about falling will inevitably limit movement among the elderly. Correspondingly, the effects on independent living ability were observed in both men and women. Thus, we should pay more attention to the safety of daily routines. In addition, regular drinking was shown to be significantly associated with IADL in men. Even if the elderly do not consume alcohol excessively and measurement of alcohol consumption is inexact, the harmful influence on the IADL score was shown. Moreover, usual alcohol consumption, rather than alcohol use disorder, has be reported to be associated with notable cognitive, social and psychological consequences in later life. ${ }^{27} 28$ Those negative effects together with our result indicated that alcohol consumption should be made with caution for the elderly. Therefore, guiding the elderly to avoid alcohol seems to be able to contribute to independent living ability.

In comparison to behavioural factors, the effects of social-psychological factors on independent living ability of the elderly were weaker with adjustment for demographic characteristics and health status. A previous study reported that social participation enhanced the ability of the elderly to live independently. ${ }^{29}$ In this study, feeling lonely and participating in entertainment were shown to be significantly associated with independent living ability, but only in men. This finding might be caused by the Chinese traditional gender-role attitude 'men go out to work and women stay at home'. This attitude indicates that men are more suitable for a job, earning money and social engagement, whereas women are more suitable for housework and taking care of family members. Even if the society has developed well in China, this gender-role attitude does not change a lot, especially for the elderly. ${ }^{30}$ Thus, even if nearly one-half of women would go out and enjoy some entertainment, the animated atmosphere seemed to be more effective in keeping men in good physical and mental health compared with women, who are accustomed to being confined to be at home.

As for the confounders, the effects have been well documented in the studies performed in China. ${ }^{10-12}$ In the current study, ethnicity was shown to be the second strongest factors associated with independent living ability among women. This ethnicity-related difference might be due to underdevelopment of social security and strong consciousness of clan and the family of minority ${ }^{31}$ which reflected the vulnerability of the elderly-care policy in minority area. Among our study population, the minority was $<5 \%$ in men. Thus, ethnic effect was not analysed among men.

There were some limitations in our study. First, the measurements of several assessed factors were too broad and simplistic, such as smoking and regular drinking. Second, even if our study was a population-based study, it was limited by the cross-sectional design. Therefore, a prospective study is required to draw any causal conclusions.

\section{CONCLUSION}

Our results showed that independent living ability among the elderly in urban areas in Liaoning Province in China is at a low level. Physical activity has an important role in maintaining and/or improving independent living ability of the elderly, even if health status has a crucial effect. Social-psychological factors, such as feeling lonely and participating in entertainment, could affect independent living ability among elderly men. Our findings suggest that gender-specific healthcare should be adopted as part of health promotion for the elderly. Physical activity of any type should be encouraged to maintain and improve the independent living ability of the elderly.

\section{Author affiliations \\ ${ }^{1}$ School of Public Health, China Medical University, Shenyang, China ${ }^{2}$ China First Mandarin Group Northeast International Hospital, Shenyang, China ${ }^{3}$ Department of Noncommunicable Chronic Disease Prevention, Liaoning Provincial Center for Disease Control and Prevention, Shenyang, China}

Contributors WS designed the study and supervised the performance of study. BW and YW collected the data, analysed the data and wrote the manuscript. TZ and JH 
helped with cleaning the data, giving advice on statistical analysis and revising the manuscript. LY coordinated data collection. All authors have given final approval of the version to be published.

Funding This research was funded by the National Natural Science Foundation of China [grant number: 81102193].

Disclaimer The funding agencies are not responsible for the opinions presented in the manuscript. The funding bodies had no influence on the conduct of the study or the interpretation of the results.

Competing interests None declared.

Patient consent for publication Obtained.

Ethics approval The study protocol and informed consent form received ethics approval from the Committee on Human Experimentation at the China Medical University. Written informed consent concerning conduct of the survey was obtained from each participant.

Provenance and peer review Not commissioned; externally peer reviewed. Data availability statement № data are available.

Open access This is an open access article distributed in accordance with the Creative Commons Attribution Non Commercial (CC BY-NC 4.0) license, which permits others to distribute, remix, adapt, build upon this work non-commercially, and license their derivative works on different terms, provided the original work is properly cited, appropriate credit is given, any changes made indicated, and the use is non-commercial. See: http://creativecommons.org/licenses/by-nc/4.0/.

\section{ORCID iD}

Wei Sun http://orcid.org/0000-0002-0241-6603

\section{REFERENCES}

1 Mai $Y$, Peng X, Chen W. How fast is the population ageing in China? Asian Popul Stud 2013;9:216-39.

2 De Luca d'Alessandro E, Bonacci S, Giraldi G. Aging populations: the health and quality of life of the elderly. Clin Ter 2011;162:e13-18.

3 Arslantas D, Ünsal A, Ozbabalık D. Prevalence of depression and associated risk factors among the elderly in middle Anatolia, Turkey. Geriatr Gerontol Int 2014;14:100-8.

$4 \mathrm{Mu} \mathrm{G}$, Zhang T. The development of population aging in China and the way to deal with it. J Huazhong Norm Univ 2011;50:29-36. (Article in Chinese).

5 Tanimoto Y, Watanabe M, Sun W, et al. Association between muscle mass and disability in performing instrumental activities of daily living (IADL) in community-dwelling elderly in Japan. Arch Gerontol Geriatr 2012;54:e230-3.

6 Lipskaya L, Jarus T, Kotler M. Influence of cognition and symptoms of schizophrenia on IADL performance. Scand J Occup Ther 2011;18:180-7.

7 Gross AL, Rebok GW, Unverzagt FW, et al. Cognitive predictors of everyday functioning in older adults: results from the active cognitive intervention trial. J Gerontol B Psychol Sci Soc Sci 2011;66B:557-66

8 Sun W, Watanabe M, Tanimoto Y, et al. Factors associated with good self-rated health of non-disabled elderly living alone in Japan: a cross-sectional study. BMC Public Health 2007;7:297.

9 Gadalla TM. Relative body weight and disability in older adults: results from a national survey. J Aging Health 2010;22:403-18.

10 RX N, Shen WZ, Bao Y, et al. Impact of the elderly chronic diseases in ability of daily life. Chin Gen Pract 2001;4:42-4. (Article in Chinese).

11 Tang GF, Wang Y, Zhao YS, et al. Study on activities of daily living and its related factors in the elderly in two cities of Anhui Province. Chin J Gerontol 2001;20:213-5. (Article in Chinese).
$12 \mathrm{Wu}$ Y, Xu Y. Investigation on daily living ability of the elderly in urban area of Suzhou City. Chin J Gerontol 2014;34:745-6. (Article in Chinese).

13 Huang S, Yuan L, Deng Y, et al. Effect of intervention of various forms of health education on health behavior of elderly patients with COPD. Med Innovat China 2013;10:49-51. (Article in Chinese).

14 Chapman KM, Ham JO, Liesen P, et al. Applying behavioral models to dietary education of elderly diabetic patients. J Nutr Educ 1995;27:75-9.

15 Seixas AA, Trinh-Shevrin C, Ravenell J, et al. Culturally tailored, peer-based sleep health education and social support to increase obstructive sleep apnea assessment and treatment adherence among a community sample of blacks: study protocol for a randomized controlled trial. Trials 2018;19:519.

16 McGrory S, Shenkin SD, Austin EJ, et al. Lawton IADL scale in dementia: can item response theory make it more informative? Age Ageing 2014;43:491-5.

17 Xue B, Jiang G, Ye G, et al. Investigation on activities of daily living and its related influential factors in retired cadres in Yangpu district, Shanghai. Chin J Prev Contr Chron Non-commun Dis 2006;14:410-2. (Article in Chinese)

18 Sun W, Aodeng S, Tanimoto Y, et al. Quality of life (QOL) of the community-dwelling elderly and associated factors: a populationbased study in urban areas of China. Arch Gerontol Geriatr 2015;60:311-6.

19 Kimura $\mathrm{Y}$, Ogawa $\mathrm{H}$, Yoshihara A, et al. Evaluation of chewing ability and its relationship with activities of daily living, depression, cognitive status and food intake in the community-dwelling elderly. Geriatr Gerontol Int 2013;13:718-25.

20 National Bureau of Statistics of China. China statistical yearbook, 2016. http://www.stats.gov.cn/tjsj/ndsj/2016/indexch.htm

21 Steiger JH. Structural model evaluation and modification: an interval estimation approach. Multivariate Behav Res 1990;25:173-80.

22 Helbostad JL, Sletvold O, Moe-Nilssen R. Home training with and without additional group training in physically frail old people living at home: effect on health-related quality of life and ambulation. Clin Rehabil 2004;18:498-508.

23 Bartlem KM, Bowman JA, Freund M, et al. Care provision to prevent chronic disease by community mental health clinicians. Am J Prev Med 2014;47:762-70.

24 Al-Hamzawi AO, Rosellini AJ, Lindberg M, et al. The role of common mental and physical disorders in days out of role in the Iraqi general population: results from the who world mental health surveys. $J$ Psychiatr Res 2014;53:23-9.

25 Kai MC, Anderson M, Lau EMC. Exercise interventions: defusing the world's osteoporosis time bomb. Bull World Health Organ 2003;81:827-30

26 De Laet CEDH, Pols HAP. Fractures in the elderly: epidemiology and demography. Best Pract Res Clin Endocrinol Metab 2000;14:171-9.

27 Yi S-W, Jung M, Kimm H, et al. Usual alcohol consumption and suicide mortality among the Korean elderly in rural communities: Kangwha cohort study. J Epidemiol Community Health 2016;70:778-83

28 Hogenkamp PS, Benedict C, Sjögren P, et al. Late-life alcohol consumption and cognitive function in elderly men. Age 2014;36:243-9.

29 Tomioka K, Kurumatani N, Hosoi H. Correction: social participation and the prevention of decline in effectance among communitydwelling elderly: a population-based cohort study. PLoS One 2016;11:e0164925

30 Trend XQ. Source and heterogeneity of the change of gender-role attitude in China: a case study of two indicators. Collect Women Stud 2016;3:33-43. (Article in Chinese).

31 Yang $\mathrm{G}$. On the aging society and related policies in ethnic regions. J Guangxi Teach Educ Univ 2016;37:53-8. 